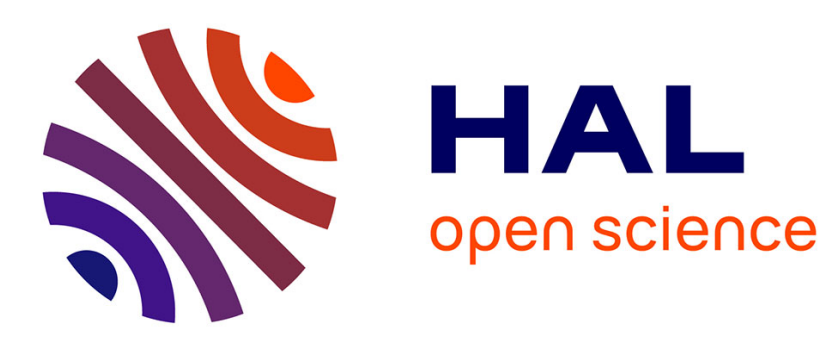

\title{
A double modal synthesis approach for brake squeal prediction
}

M Monteil, S Besset, Jean-Jacques Sinou

\section{To cite this version:}

M Monteil, S Besset, Jean-Jacques Sinou. A double modal synthesis approach for brake squeal prediction. Mechanical Systems and Signal Processing, 2016, 70-71, pp.1073 - 1084. 10.1016/j.ymssp.2015.07.023 . hal-03258001

\section{HAL Id: hal-03258001 \\ https://hal.science/hal-03258001}

Submitted on 11 Jun 2021

HAL is a multi-disciplinary open access archive for the deposit and dissemination of scientific research documents, whether they are published or not. The documents may come from teaching and research institutions in France or abroad, or from public or private research centers.
L'archive ouverte pluridisciplinaire $\mathbf{H A L}$, est destinée au dépôt et à la diffusion de documents scientifiques de niveau recherche, publiés ou non, émanant des établissements d'enseignement et de recherche français ou étrangers, des laboratoires publics ou privés. 
M. Monteil, S. Besset and J-J. Sinou, A double modal synthesis approach for brake squeal prediction, Mechanical Systems and Signal Processing, 70-71, 1073-1084, 2016.

doi:10.1016/j.ymssp.2015.07.023

\title{
A double modal synthesis approach for brake squeal prediction
}

\author{
M. Monteil ${ }^{a, d}$, S. Besseta ${ }^{a, b, e}$, J.-J. Sinou ${ }^{a, b, c, f}$ \\ ${ }^{a}$ Laboratoire de Tribologie et Dynamique des Systèmes, UMR CNRS 5513, École Centrale de Lyon, 36 \\ avenue Guy de Collongue 69134 Écully Cedex, France \\ ${ }^{b}$ Centre Lyonnais d'Acoustique, Université de Lyon, 69622 Lyon, France \\ ${ }^{c}$ Institut Universitaire de France, 75005 Paris, France \\ ${ }^{d}$ melodie.monteil@gmail.com \\ esebastien.besset@ec-lyon.fr

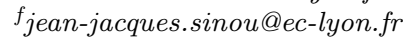

\begin{abstract}
This paper is devoted to propose a new efficient reduction method for predicting the stability analysis of a brake system subjected to friction-induced vibration. The finite element brake system under study is composed of a disc and a pad. The contact is modeled by introducing contact elements at the friction interface with the classical Coulomb law and a constant friction coefficient. It will be demonstrated that it is possible to build efficient reduced finite element models by developing a reduced model based on a Double Modal Synthesis (i.e. a classical modal reduction via Craig \& Bampton plus a condensation at the frictional interface). Special attention is being conducted to validate the convergence of the reduced model especially on the approximation of the unstable modes with respect to real and imaginary parts. This complete numerical strategy based on Double Modal Synthesis allows us to perform relevance squeal prediction of unstable vibration modes. It is demonstrated that the numerical results via the Double Modal Synthesis are in good agreement with those of the classical Craig \& Bampton method. Keywords: friction-induced vibration, stability analysis, reduction strategy, squeal
\end{abstract}

\section{Introduction}

Research for predicting squeal noise has been regularly performed for many years [1, 2, 3. Despite great progress in the understanding and numerical simulation of brake squeal (i.e. methodology for the modeling of the variability [6, 7], damping effects on squeal

5 [4, 5], statistical analysis of brake squeal noise [8], non-linear formulations and behaviors 
at the frictional interfaces $[9,10,11,12$, chaotic phenomenon [13] or acoustic emissions [14, 15, 16]), there is still considerable progress to provide in order to achieve efficient numerical approaches for squeal prediction. One of the most important drawbacks that needs to be investigated is the capability to reduce computational time and data storage.

One of the possible ways is to propose minimal or reduced finite element models that can reproduce the propensity of squeal noise. In this work, the focus is on an original numerical modal reduction in order to predict the stability analysis of large finite element models for brake systems. Indeed, it appears that one of the major limitations of classical reductions for squeal noise is associated with the size of interface matrices due to the ${ }_{15}$ explicit use of the interface degrees of freedom (i.e. the size of the reduced finite element model is strongly dependent on the number of the degrees of freedom at the frictional interface). So an interesting concept should be to develop an implicit reduction at the frictional interface in order to return accurate results on stability analysis by keeping a minimal number of generalized degrees of freedom at the interface. This reduction (at 20 the frictional interface) in connection with conventional reductions (for each of the substructures) must be able to reproduce the overall behavior of the system for the prediction of the squeal propensity. In this work, we propose a new original strategy based on the Double Modal Synthesis [17, 18]: the reduced finite element model is performed by using the combination of the Craig \& Bampton reduction procedure [19] with an interface 25 reduction that allows to reduce the size of the internal interface between substructures.

This paper is divided into four parts: firstly, the finite element model under study for brake squeal is presented with the modeling hypotheses. Secondly, the classical Craig \& Bampton reduction is briefly discussed and the the relevance of this reduction method for the stability analysis of the proposed finite element model is studied in details. The last 30 part is devoted to the presentation of the original proposed strategy based on the Double Modal Synthesis. Finally, discussion of numerical results is proposed. A comparison with the classical Craig \& Bampton method (without reduction at the frictional interface) is carried out with an evaluation of the computational performances. 


\section{The model of the simplified brake system}

35 This section is firstly devoted to present the finite element model of the simplified brake system under study. Secondly, the classical stability analysis for the complete system that will served as a reference for the following parts of the paper is discussed.

\subsection{Finite element model}

The finite element model under study corresponds to a simplified brake system that

40 is composed of two isotropic elastic structures: a circular disc and a pad [12, 20]. The Structural Dynamics Toolbox (SdTools - Matlab Software) is used to build the finite element model of the two substructures as shown in Figure 1 . Concerning the boundary conditions, the pad is in-plane fixed and the inner surface of the disc is clamped. Details on the finite element model and material properties, inspired from [15], are listed in Table

$45 \quad 1$ and 2 respectively.

[Figure 1 about here.]

[Table 1 about here.]

[Table 2 about here.]

The equations of motion of the brake system can be written as

$$
\mathbf{M} \ddot{\mathbf{X}}+\mathbf{C} \dot{\mathbf{X}}+\mathbf{K X}=\mathbf{F}+\mathbf{F}_{\mathbf{p}}
$$

where $\mathbf{M}, \mathbf{C}, \mathbf{K}$ are the mass, damping and stiffness matrices, respectively. $\ddot{\mathbf{X}}, \dot{\mathbf{X}}, \mathbf{X}$ are acceleration, velocity and displacement vectors. $\mathbf{F}_{\mathbf{p}}$ defines the vector of the pressure force. $\mathbf{F}$ is related to the vector of forces due to contact and friction occurring at the disc/ pad interface especially at the contact nodes. The contact force is described by the following mathematical function

$$
F_{c}^{i}=k_{L}\left(z_{p}-z_{d}\right)
$$

where $z_{p}$ and $z_{d}$ define, respectively, the displacements of contact nodes for the pad and the disc at the $i^{t h}$ contact element. $k_{L}$ is the contact stiffness coefficient fixed at $3.1 \times$ $10^{7} \mathrm{~N} . \mathrm{m}^{-1}$. This value has been chosen to fit the first order of pad compression 
curves obtained from experimental tests given in [20]. This value is only valuable over a predefined pressure range. Moreover, a simplified Coulomb law is considered with a constant friction coefficient without any stick-slip motion. Then, the friction force $F_{f}^{i}$, located at the $i^{\text {th }}$ node is derived from the contact force $F_{c}^{i}$ at the friction interface in the tangential plan with

$$
F_{f}^{i}=\mu F_{c}^{i}
$$

where $\mu$ is the friction coefficient.

Thus, the vector of friction force is given by

$$
\mathbf{F}_{\mathbf{f}}=\begin{aligned}
& \mu F_{c, z} \mathbf{e}_{\theta} \cdot \mathbf{x} \\
& \mu F_{c, z} \mathbf{e}_{\theta} \cdot \mathbf{y}
\end{aligned}
$$

where $\mathbf{x}$ and $\mathbf{y}$ are the tangential directions of the friction interface, $\mathbf{e}_{\theta}$ is the orthoradial direction of the disc. The vector of the non-linear force is composed of the contact and contact components $\left(\mathbf{F}_{\mathbf{c}}\right)$ and $\left(\mathbf{F}_{\mathbf{f}}\right)$, respectively (i.e. $\mathbf{F}=\mathbf{F}_{\mathbf{c}}+\mathbf{F}_{\mathbf{f}}$ ). Finally, modal damping associated with each eigenfrequency is applied to the system as $c_{k}=2 \xi_{k} \omega_{k}$, where $\xi_{k}$ is the damping coefficient. The value of the damping coefficient $\xi_{k}$ is fixed at $2 \%$. Even if the effects of damping is out the scope of the present study, we recall that damping proves to have two different effects: a lowering effect and a smoothing effect. The lowering effect tends to stabilize the system. If the smoothing effect prevails, added damping may destabilize the system see the following paper for more details [5, 22, 23, 24, 25]. The damping matrix $\mathbf{C}$ is built expanding the modal damping matrix onto the undamped, non-frictional inverse modal basis $\boldsymbol{\Phi}^{-1}$ of the reduced model as

$$
\mathbf{C}=\boldsymbol{\Phi}^{-1 T} \operatorname{diag}\left[c_{1} \cdots c_{k} \cdots c_{N}\right] \boldsymbol{\Phi}^{-1} .
$$

Note that the assembled finite element model is composed of 44950 degrees of freedom.

\subsection{Stability analysis}

The stability analysis is a classic process in order to study mechanical system subjected to friction-induced vibration. The first step called the static problem consists of estimating the steady-state operating point $\mathbf{X}_{\mathbf{0}}$ for the full set of static equations. Then, stability is performed on the linearized equations for small perturbations at the operating 
point $\left(\mathbf{X}=\mathbf{X}_{\mathbf{0}}+\overline{\mathbf{X}}\right)$. So, the system around the operating point is given by

$$
\mathbf{M} \ddot{\overline{\mathbf{X}}}+\mathbf{C} \dot{\overline{\mathbf{X}}}+\underbrace{(\mathbf{K}-\mathbf{J})}_{\mathcal{K}} \overline{\mathbf{X}}=\mathbf{0},
$$

where $\mathbf{J}$ is the frictional contact Jacobian matrix derived from the expressions of the contact and friction forces.

The associated eigenvalue problem can be written as

$$
\left(\lambda^{2} \mathbf{M}+\lambda \mathbf{C}+\mathcal{K}\right) \mathbf{\Phi}=\mathbf{0},
$$

where $\lambda_{k}$ and $\Phi_{k}$ are the eigenvalue and eigenvector related to the $k^{t h}$ eigenmode. As the stiffness matrix $\mathcal{K}$ is asymmetrical, due to the contribution of friction forces, the computed eigenvalues are complex and ca be written as

$$
\lambda_{k}=a_{k}+i \omega_{k}
$$

where $a_{k}$ is the real part and $\omega_{k}$ is the imaginary part of the eigenvalue $\lambda_{k} \cdot \omega_{k}$ corresponds to the pulsation of the $k^{t h}$ mode. As long as the real part of all the eigenvalues remains

${ }_{55}$ negative, the system is stable. When at least one of the eigenvalues has a positive real part, the system becomes unstable. The unstable mode can generate vibrations at its natural frequency with coalescence phenomenon, resulting in squeal noise emission. The frequency of the unstable mode is given by the imaginary part of this eigenvalue.

\section{Craig \& Bampton reduction}

The main objective of this section is to discuss the relevance of the $\mathrm{C} \& \mathrm{~B}$ reduction method for the stability analysis of the proposed disc/pad system. More specifically, the analysis will focus on the possibility to reduce the size of the full finite element model while maintaining an accurate estimation of the evolution of the imaginary and real parts of the system with a low order model. First of all, the $\mathrm{C} \& \mathrm{~B}$ reduction method is

${ }_{65}$ briefly presented. Then, performances of the $\mathrm{C} \& \mathrm{~B}$ reduction methods are discussed on the frictionless problem and the problem with friction at the pad/disc interface.

\subsection{CESB condensation method}

The $\mathrm{C} \& \mathrm{~B}$ reduction method consists in building a projection base combining constraint modes and a truncated basis of normal modes computed with a fixed interface. 
70 The truncation is realized on each substructure of the brake system (i.e. the pad and the disc). All the degrees of freedom (denoted by $\mathbf{u}$ ) are divided in two types: the subsystem internal degrees of freedom (denoted by $\mathbf{u}_{\mathbf{i}}$ ) and the boundary degrees of freedom (denoted by $\mathbf{u}_{\mathbf{j}}$ ). So the degrees of freedom at the frictional interface are directly associated with the boundary degrees of freedom.

${ }_{75}$ In this section, we consider a single substructure (either the pad or the disc). Mass and stiffness matrices related to the considered substructure are denoted using the superscripts $D$ (disc) or $P$ (pad), as well as the degrees of freedom. Hence the following formulation:

$$
\begin{aligned}
\mathbf{u} & =\left\{\begin{array}{c}
\mathbf{u}_{\mathbf{j}}{ }^{P} \\
\mathbf{u}_{\mathbf{i}}{ }^{P} \\
\mathbf{u}_{\mathbf{j}}{ }^{D} \\
\mathbf{u}_{\mathbf{i}}{ }^{D}
\end{array}\right\} \\
\mathbf{K} & =\left[\begin{array}{cccc}
\mathbf{K}_{\mathbf{j} \mathbf{j}}{ }^{P} & \mathbf{K}_{\mathbf{j i}}{ }^{P} & \mathbf{0} & \mathbf{0} \\
\mathbf{K}_{\mathbf{j i}}{ }^{P} & \mathbf{K}_{\mathbf{i i}}{ }^{P} & \mathbf{0} & \mathbf{0} \\
\mathbf{0} & \mathbf{0} & \mathbf{K}_{\mathbf{j} \mathbf{j}}{ }^{D} & \mathbf{K}_{\mathbf{j i}}{ }^{D} \\
\mathbf{0} & \mathbf{0} & \mathbf{K}_{\mathbf{j i}}{ }^{D} & \mathbf{K}_{\mathbf{i i}}{ }^{D}
\end{array}\right] \\
\mathbf{M} & =\left[\begin{array}{cccc}
\mathbf{M}_{\mathbf{j} \mathbf{j}}{ }^{P} & \mathbf{M}_{\mathbf{j i}}{ }^{P} & \mathbf{0} & \mathbf{0} \\
\mathbf{M}_{\mathbf{j i}}{ }^{P} & \mathbf{M}_{\mathbf{i i}}{ }^{P} & \mathbf{0} & \mathbf{0} \\
\mathbf{0} & \mathbf{0} & \mathbf{M}_{\mathbf{j} \mathbf{j}}{ }^{D} & \mathbf{M}_{\mathbf{j i}}{ }^{D} \\
\mathbf{0} & \mathbf{0} & \mathbf{M}_{\mathbf{j i}}{ }^{D} & \mathbf{M}_{\mathbf{i i}}{ }^{D}
\end{array}\right]
\end{aligned}
$$

The $\mathrm{C} \& \mathrm{~B}$ reduction aims at expressing the internal degrees of freedom $\mathbf{u}_{\mathbf{i}}{ }^{q}$, where $q=P$ or $D$, as a function of generalized degrees of freedom $\boldsymbol{\eta}^{q}$ and boundary degrees of freedom $\mathbf{u}_{\mathbf{j}}^{q}$ :

$$
\mathbf{u}_{\mathbf{i}}^{q}=\boldsymbol{\Phi}^{q} \eta^{q}+\boldsymbol{\Psi}^{q} \mathbf{u}_{\mathbf{j}}^{q}
$$

The columns of matrix $\boldsymbol{\Phi}^{q}$ are the eigenvectors $\boldsymbol{\Phi}_{n}{ }^{q}$ obtained by solving the following so equation:

$$
\left(\mathbf{K}_{\mathbf{i i}}{ }^{q}-\omega_{n}^{2} \mathbf{M}_{\mathbf{i i}}{ }^{q}\right) \Phi_{n}{ }^{q}=\mathbf{0}
$$


Matrix $\boldsymbol{\Psi}$ is obtained by solving the following static problem:

$$
\mathbf{K}_{\mathbf{i i}}{ }^{q} \mathbf{u}_{\mathbf{i}}{ }^{q}+\mathbf{K}_{\mathbf{i j}}{ }^{q} \mathbf{u}_{\mathbf{j}}{ }^{q}=\mathbf{0} \quad \Rightarrow \quad \mathbf{u}_{\mathbf{i}}^{q}=\underbrace{-\left(\mathbf{K}_{\mathbf{i i}}{ }^{q}\right)^{-1} \mathbf{K}_{\mathbf{i j}}{ }^{q}}_{\Psi} \mathbf{u}_{\mathbf{j}}{ }^{q}
$$

Matrices $\boldsymbol{\Psi}$ and $\boldsymbol{\Phi}$ allow to express the degrees of freedom as follows:

$$
\mathbf{u}=\left\{\begin{array}{c}
\mathbf{u}_{\mathbf{j}}{ }^{P} \\
\mathbf{u}_{\mathbf{i}}{ }^{P} \\
\mathbf{u}_{\mathbf{j}}{ }^{D} \\
\mathbf{u}_{\mathbf{i}}{ }^{D}
\end{array}\right\}=\underbrace{\left[\begin{array}{cccc}
\mathbf{I} & \mathbf{0} & \mathbf{0} & \mathbf{0} \\
\boldsymbol{\Psi}^{P} & \boldsymbol{\Phi}^{P} & \mathbf{0} & \mathbf{0} \\
\mathbf{0} & \mathbf{0} & \mathbf{I} & \mathbf{0} \\
\mathbf{0} & \mathbf{0} & \boldsymbol{\Psi}^{D} & \boldsymbol{\Phi}^{D}
\end{array}\right]}_{\mathbf{T}}\left\{\begin{array}{c}
\mathbf{u}_{\mathbf{j}}{ }^{P} \\
\boldsymbol{\eta}^{P} \\
\mathbf{u}_{\mathbf{j}}{ }^{D} \\
\boldsymbol{\eta}^{D}
\end{array}\right\}
$$

The reduced mass and stiffness matrices can then be written:

$$
\tilde{\mathbf{M}}=\mathbf{T}^{T} \mathbf{M T}
$$

$$
\tilde{\mathbf{K}}=\mathbf{T}^{T} \mathbf{K} \mathbf{T}
$$

$\left[\boldsymbol{\eta}^{D}\right]$ and $\left[\boldsymbol{\eta}^{P}\right]$ become the two control parameters of the C\&B condensation. As the

85 number of junction dof's is the same for each substructure, consequently the expression $\left[\mathbf{u}_{\mathbf{j}}{ }^{P}\right]=\left[\mathbf{u}_{\mathbf{j}}{ }^{D}\right]$ is verified. Finally it can be concluded that the C\&B reduction leads to a low order system depending on the number of contact nodes and the number of modes kept in the truncation of the two subsystems independently. More precisely, as we can find three dof's per contact node (because of the three directions) $\left[\mathbf{u}_{\mathbf{j}}{ }^{P}\right]=\left[\mathbf{u}_{\mathbf{j}}{ }^{D}\right]=3 \times n_{c}$

90 where $n_{c}$ defines the number of contact nodes $\left(n_{c}=200\right.$ in the case under study). So that the size of the $\mathrm{C} \& \mathrm{~B}$ reduced model is finally obtained by the following relationship $2 \times 3 \times n_{c}+\left[\boldsymbol{\eta}^{P}\right]+\left[\boldsymbol{\eta}^{D}\right]$.

\subsection{Performances of the Craig \& Bampton reduction}

Before using reduced bases for the stability study, performances of the C\&B reduction method has to be tested on the problem without friction. So in this following part of this section, $\mathrm{C} \& \mathrm{~B}$ reduction is applied to the mechanical system under study for two specific problems. The first case concerns the system without friction at the disc/pad interface (i.e. the disc and the pad are coupled only through normal degrees of freedom 
on the contact surface). The second case introduces the problem with friction and the associated stability analysis. The system without friction at the disc/pad interface (i.e. the disc and the pad are coupled only through normal degrees of freedom on the contact surface).

For each case (i.e. the frictionless problem and the problem with friction), the objective consists in searching for the best approximated solution in a reduced subspace for the brake system:

$$
\left(\tilde{\lambda}^{2} \tilde{\mathbf{M}}+\tilde{\lambda} \tilde{\mathbf{C}}+\tilde{\mathcal{K}}\right) \tilde{\boldsymbol{\Phi}}=\mathbf{0}
$$

where $\tilde{\mathbf{M}}$, $\tilde{\mathbf{C}}$ and $\tilde{\mathcal{K}}$ correspond to the mass, damping and stiffness matrices in the reduced subspace. For the reader comprehension, the change between the two cases comes exclusively from the expression of the stiffness matrix which includes or not the friction terms.

By solving the previous reduced eigenvalue problem (equation (18)), a set of approximate complex eigenvalues $\tilde{\lambda}_{k}=\tilde{a}_{k}+i \tilde{\omega}_{k}$ can be estimated in the frequency range of interest. Due to the fact that the aim of this section is to study the convergence of the reduced model in regard to the number of modes used in the $\mathrm{C} \& \mathrm{~B}$ reduction, two criteria employed to evaluate the quality of the $\mathrm{C} \& \mathrm{~B}$ reduction method are proposed. For the frictionless problem, these criteria are based on the relative error criterion between imaginary and real parts for each mode in the frequency range of interest $[0 ; 6000] \mathrm{Hz}$. Error on the frequencies is defined by the following relation

$$
\varepsilon_{\omega, k}=100 \frac{\omega_{k}-\tilde{\omega}_{k}}{\omega_{k}},
$$

where $\omega_{k}$ is the exact pulsation of the $k^{t h}$ mode (i.e. pulsation of the original full brake model) and $\tilde{\omega}_{k}$ is the approximate pulsation of the $k^{t h}$ mode calculated via the C\&B reduction.

The calculation of error on the real parts is given by

$$
\varepsilon_{a, k}=100 \frac{a_{k}-\tilde{a}_{k}}{a_{k}},
$$

where $a_{k}$ and $\tilde{a}_{k}$ are the exact and approximate real parts of the $k^{t h}$ mode, respectively.

For the problem with friction, the criteria are only based on the relative error criterion between imaginary and real parts for the unstable modes in the frequency range of interest 
$[0 ; 6000] \mathrm{Hz}$. Error on the frequencies for the $k^{\text {th }}$ unstable mode is defined by the following relation

$$
\varepsilon_{\omega, k}^{u}=100 \frac{\omega_{k}^{u}-\tilde{\omega}_{k}^{u}}{\omega_{k}^{u}}
$$

where $\omega_{k}^{u}$ is the exact pulsation of the $k^{t h}$ unstable mode (i.e. pulsation of the original full brake model) and $\tilde{\omega}^{u}{ }^{u}$ is the approximate pulsation of the $k^{\text {th }}$ unstable mode calculated via the $\mathrm{C} \& \mathrm{~B}$ reduction.

The calculation of error on the real parts (i.e. divergence rate) of the $k^{\text {th }}$ unstable mode is given by

$$
\varepsilon_{a, k}^{u}=100 \frac{a_{k}^{u}-\tilde{a}_{k}^{u}}{a_{k}^{u}}
$$

105 where $a_{k}{ }^{u}$ and $\tilde{a}_{k}^{u}$ are the exact and approximate real parts of the $k^{t h}$ unstable mode, respectively.

First of all, results for the frictionless problem are presented. This analysis is a classical problem in the field of mechanical engineering. However it is presented here as a preliminary study to the specific formalism used further in the stability study. Figure 2 shows the errors $\varepsilon_{\omega, k}$ and $\varepsilon_{a, k}$ on the frequencies and real parts in the frequency range of interest $[0 ; 6000] \mathrm{Hz}$ for different sizes of the reduced subspace. It clearly appears that a reduced subspace composed of 40 internal modes is enough to stay under an error of $0.4 \%$ for eigenfrequencies.

Secondly, Figures 3 and 4 show the convergence results on both real and imaginary parts of the three unstable modes in the frequency range of interest in the case of $\mu=0.5$ and $\mu=1$ (for the problem with friction at the disc/pad interface). As previously explained, we focus our interest only on the unstable modes in the frequency range of interest. This choice results in the fact that for a stability analysis, it is particularly interesting to correctly predict the squeal occurrence and frequencies and the real parts of the associated unstable modes. The results of convergence for the stable modes (not presented here) are similar.

Even if it is shown that the errors $\varepsilon_{\omega, k}$ and $\varepsilon_{a, k}$ remain under $1 \%$ for a sufficient size of the reduced space, it is observed that the real parts of the three unstable modes converge slower than the associated imaginary parts for both $\mu=0.5$ and $\mu=1$. We can also note that the size of the reduced base required to achieve a given level of error 
is smaller for the the imaginary parts than for the real parts (for both cases $\mu=0.5$ and $\mu=1)$.

[Figure 2 about here.]

[Figure 3 about here.]

\section{Double Modal Synthesis}

Even if the $\mathrm{C} \& \mathrm{~B}$ reduction procedure allows to reduce the size of the system, it can be noted that one of the major limitations of this reduction is associated with the size of interface matrices due to the explicit use of the degrees of freedom at the interface

135 (in the case under study the 220 contact nodes lead to 1320 degrees of freedom). As a consequence, the size of the reduced system is strongly dependent on the number of degrees of freedom at the frictional interface. So it should be interesting to propose a global strategy that generates an implicit reduction at the frictional interface in order to return accurate results for a drastically reduced finite element system in a lesser time while keeping the global physical behaviour at the frictional interface. Therefore, this section is devoted to present the combination of the $\mathrm{C} \& \mathrm{~B}$ reduction procedure with an interface reduction strategy that allows to reduce the size of the internal interface between substructures. Firstly, the reduction method at the frictional interface is presented and discussed. Then, efficiency and advantages of the global reduction are analyzed.

145 4.1. The interface reduction strategy

The Craig \& Bampton reduction explained in section 3.1 leads to reduced mass and stiffness matrices $\tilde{\mathbf{M}}$ and $\tilde{\mathbf{K}}$, as well as a reduced dof's vector $\tilde{\mathbf{u}}$ :

$$
\tilde{\mathbf{M}}=\left[\begin{array}{cccc}
\tilde{\mathbf{M}}_{\mathbf{j} \mathbf{j}}^{P} & \tilde{\mathbf{M}}_{\mathbf{j} \eta}^{P} & \mathbf{0} & \mathbf{0} \\
\tilde{\mathbf{M}}_{\eta \mathbf{j}}^{P} & \tilde{\mathbf{M}}_{\eta \eta}^{P} & \mathbf{0} & \mathbf{0} \\
\mathbf{0} & \mathbf{0} & \tilde{\mathbf{M}}_{\mathbf{j} \mathbf{j}}^{D} & \tilde{\mathbf{M}}_{\mathbf{j} \eta}^{D} \\
\mathbf{0} & \mathbf{0} & \tilde{\mathbf{M}}_{\eta \mathbf{j}}^{D} & \tilde{\mathbf{M}}_{\eta \eta}^{D}
\end{array}\right]
$$




$$
\begin{gathered}
\tilde{\mathbf{K}}=\left[\begin{array}{cccc}
\tilde{\mathbf{K}}_{\mathbf{j} \mathbf{j}}^{P} & \tilde{\mathbf{K}}_{\mathbf{j} \eta}^{P} & \mathbf{0} & \mathbf{0} \\
\tilde{\mathbf{K}}_{\eta \mathbf{j}}^{P} & \tilde{\mathbf{K}}_{\eta \eta}^{P} & \mathbf{0} & \mathbf{0} \\
\mathbf{0} & \mathbf{0} & \tilde{\mathbf{K}}_{\mathbf{j} \mathbf{j}} & \tilde{\mathbf{K}}_{\mathbf{j} \eta}^{D} \\
\mathbf{0} & \mathbf{0} & \tilde{\mathbf{K}}_{\eta \mathbf{j}}^{D} & \tilde{\mathbf{K}}_{\eta \eta}^{D}
\end{array}\right] \\
\tilde{\mathcal{K}}=\left[\begin{array}{cccc}
\tilde{\mathcal{K}}_{\boldsymbol{j} j}{ }^{P} & \tilde{\mathcal{K}}_{\boldsymbol{j} \eta}{ }^{P} & \tilde{\mathcal{K}}_{\boldsymbol{j} \boldsymbol{j}}{ }^{P D} & \mathbf{0} \\
\tilde{\mathcal{K}}_{\eta j}{ }^{P} & \tilde{\mathcal{K}}_{\boldsymbol{\eta} \boldsymbol{}}{ }^{P} & \mathbf{0} & \mathbf{0} \\
\tilde{\mathcal{K}}_{\boldsymbol{j} \boldsymbol{j}}{ }^{D P} & \mathbf{0} & \tilde{\boldsymbol{K}}_{\boldsymbol{j} \boldsymbol{j}}{ }^{D} & \tilde{\boldsymbol{K}}_{\boldsymbol{j} \boldsymbol{\eta}}{ }^{D} \\
\mathbf{0} & \mathbf{0} & \tilde{\boldsymbol{K}}_{\boldsymbol{\eta} \boldsymbol{j}}{ }^{D} & \tilde{\mathcal{K}}_{\boldsymbol{\eta} \boldsymbol{\eta}}{ }^{D}
\end{array}\right] \\
\tilde{\mathbf{u}}=\left\{\begin{array}{c}
\mathbf{u}_{\mathbf{j}}{ }^{P} \\
\boldsymbol{\eta}^{P} \\
\mathbf{u}_{\mathbf{j}}{ }^{D} \\
\boldsymbol{\eta}^{D}
\end{array}\right\}
\end{gathered}
$$

The aim of the DMS method is to express the remaining degrees of freedom $\mathbf{u}_{\mathbf{j}}{ }^{P}$ and $\mathbf{u}_{\mathbf{j}}{ }^{D}$ in function of generalized degrees of freedom $\boldsymbol{\zeta}$ :

$$
\left\{\begin{array}{c}
\mathbf{u}_{\mathbf{j}}{ }^{P} \\
\mathbf{u}_{\mathbf{j}}{ }^{D}
\end{array}\right\}=\boldsymbol{\Phi}_{b} \boldsymbol{\zeta}=\left[\begin{array}{c}
\mathbf{\Phi}_{\boldsymbol{b}}{ }^{P} \\
\boldsymbol{\Phi}_{\boldsymbol{b}}{ }^{D}
\end{array}\right] \boldsymbol{\zeta}
$$
problem:

$$
\left(\left[\begin{array}{cc}
\tilde{\mathcal{K}}_{j j}{ }^{P} & \tilde{\mathcal{K}}_{j j}{ }^{P D} \\
\tilde{\mathcal{K}}_{j \boldsymbol{j}}{ }^{D P} & \tilde{\mathcal{K}}_{\boldsymbol{j} j}{ }^{D}
\end{array}\right]-\omega_{k}^{2}\left[\begin{array}{cc}
\tilde{\mathbf{M}}_{j j}^{P} & \mathbf{0} \\
\mathbf{0} & \tilde{\mathbf{M}}_{j j}^{D}
\end{array}\right]\right) \boldsymbol{\Phi}_{\boldsymbol{b}}{ }^{q}=\mathbf{0}
$$

Vector $\tilde{\mathbf{u}}$ can now be expressed as follows:

$$
\tilde{\mathbf{u}}=\left\{\begin{array}{c}
\mathbf{u}_{\mathbf{j}}{ }^{P} \\
\boldsymbol{\eta}^{P} \\
\mathbf{u}_{\mathbf{j}}{ }^{D} \\
\boldsymbol{\eta}^{D}
\end{array}\right\}=\underbrace{\left[\begin{array}{ccc}
\boldsymbol{\Phi}_{\boldsymbol{b}}{ }^{P} & \mathbf{0} & \mathbf{0} \\
\mathbf{0} & \mathbf{I} & \mathbf{0} \\
\boldsymbol{\Phi}_{\boldsymbol{b}}{ }^{D} & \mathbf{0} & \mathbf{0} \\
\mathbf{0} & \mathbf{0} & \mathbf{I}
\end{array}\right]}_{\mathbf{T}_{\mathbf{b}}}\left\{\begin{array}{c}
\boldsymbol{\zeta} \\
\boldsymbol{\eta}^{P} \\
\boldsymbol{\eta}^{D}
\end{array}\right\}
$$


The transfer matrix $\mathbf{T}_{\mathbf{b}}$ allows to obtain the reduced mass, damping and stiffness matrices:

$$
\begin{gathered}
\hat{\mathbf{M}}=\mathbf{T}_{\mathbf{b}}{ }^{T} \tilde{\mathbf{M}} \mathbf{T}_{\mathbf{b}} \\
\hat{\mathbf{C}}=\mathbf{T}_{\mathbf{b}}{ }^{T} \tilde{\mathbf{C}} \mathbf{T}_{\mathbf{b}} \\
\hat{\mathbf{K}}=\mathbf{T}_{\mathbf{b}}{ }^{T} \tilde{\mathbf{K}} \mathbf{T}_{\mathbf{b}} \quad \text { and } \hat{\mathcal{K}}=\mathbf{T}_{\mathbf{b}}{ }^{T} \tilde{\mathcal{K}} \mathbf{T}_{\mathbf{b}}
\end{gathered}
$$

Therefor, the stability of the DMS-reduced model can be computed using the following equation:

$$
\left(\hat{\mathcal{K}}+\hat{\lambda} \hat{\mathbf{C}}+\hat{\lambda}^{2} \hat{\mathbf{M}}\right) \hat{\boldsymbol{\Phi}}=\mathbf{0}
$$

\subsection{Stability analysis for $\mu=[0 ; 1]$}

The stability of the DMS reduced model is now computed for a coefficient of friction ranging from $\mu=0$ to $\mu=1$. Several orders of truncation of the DMS are tested in order to validate the efficiency of the proposed methodology.

Figure 5 shows the evolution of the real parts of the three unstable modes versus the friction coefficient for different orders of truncation (50, 100, 150 and 200 generalized degrees of freedom). Then, Figures 6,7 and 8 give the evolution of both the real and imaginary parts in the complex plane for the first, second and third unstable modes respectively (in these cases, the friction coefficient varies in the range $\mu=[0 ; 1]$ ). It is observed that increasing the order of truncation for the DMS leads to a better estimation of the real and imaginary parts of the three complex eigenvalues for all the values of the friction coefficient in the range of interest (i.e. $\mu=[0 ; 1]$ ). These results clearly indicate the efficiency of the proposed DMS.

$\mathrm{n}$ order to undertake more precisely the convergence of the DMS-reduced model, convergence on the real and imaginary parts is given in Figures9, and 10 for two coefficients of friction $\mu=0.5$ and $\mu=1$, respectively. More specifically, errors between the DMS-reduced model and the reference model are shown versus the orders of truncation of the DMS. First of all, it is illustrated that increasing the size of the generalized degree of 
freedom for the DMS-reduced model decreases errors for both imaginary and real parts. This reflects the convergence of the DMS. Moreover, it can be noted that the errors made on the imaginary parts are smaller than the errors on the real parts regardless of the size of the DMS-reduced model.

On the other hand, the truncation order needed to achieve convergence (in terms of permissible error) is strongly dependent on the value of the friction coefficient. In order to achieve an effective reduction for the entire frequency range of interest, the DMS must be initially validated for various coefficients of friction. So considering only a validation for $\mu=0$ (i.e. without contribution of the frictional elements) will not be sufficient in order to ensure a reduced-model validated for $\mu \neq 0$.

[Figure 5 about here.]

[Figure 6 about here.]

[Figure 7 about here.]

[Figure 8 about here.]

[Figure 10 about here.]

\section{C\&B vs DMS: computation times}

In this section, computation times for both the C\&B reduction and the DMS are compared. Figure 11 presents the associated results. For the reader comprehension, the number of generalized degrees of freedom for the DMS is fixed as $[\varphi]=100$ and the C\&B order is increased as $\left[\boldsymbol{\eta}^{P}\right]=\left[\boldsymbol{\eta}^{D}\right]$.

The first column of the tabular shown in the Figure 11(a) gives several values taken by $[\boldsymbol{\eta}]$. The second column shows the C\&B-time computation and the related curve is plotted in black color on Figure 11(b). The third column of the tabular shown in the Figure 11(a) gives DMS-computation time and the associated curve is plotted in gray 
color on Figure 11(b). It is clearly observed that the DMS greatly reduces computational times because of the weak number of generalized degrees of freedom kept in the truncation.

[Figure 11 about here.]

\section{Conclusion}

This paper undertakes two modal reduction methods (the Craig \& Bampton reduction and the Double Modal Synthesis) with an application on a finite element model of brake squeal. More specifically, the Double Modal Synthesis is described and the efficiency of this proposed reduction strategy is illustrated via the stability analysis of brake squeal. It appears that one of the most advantages of the Double Modal Synthesis is to be able to drastically reduce the number of degree of freedom of the system by performing a modal synthesis at the frictional interface. Numerical results also indicate that the size of the final system via the Double Modal Synthesis strongly depends on the values of some physical parameters (such as the friction coefficient). It is also illustrated that considering a modal reduction only based on the frictionless system (for $\mu=0$ ) can lead to spurious results. In all cases, the efficiency and convergence of the proposed Double Modal Synthesis is validated.

In this present study, we consider the friction coefficient to be a parameter and the modes are computed for specific values of the friction coefficient $\mu$, which could lead to high computational costs. In future work, improvements of the proposed second condensation should be based on complex modes computed for different values of the friction coefficient. This could lead to a generalization of the reduction over a variation range of the friction coefficient.

\section{Acknowledgments}

The authors would like to thank the financial support provided by the French Na-tional Research Agency through the framework of its project ANR-12-JS09-0009. This work was achieved within Stellab program - OpenLab Vibro-AcousticTribology@Lyon(VAT@Lyon).

J-J. Sinou acknowledges the support of the Institut Universitaire de France. 


\section{References}

[1] N.M. Kinkaid, O.M. O'Reilly, and P. Papadopoulos. Automotive disc brake squeal. Journal of Sound and Vibration, 267:105-166, 2003.

[2] R.A. Ibrahim. Friction-induced vibration, chatter, squeal, and chaos part 1: mechanics of contact and friction. Am Soc Mech Eng Appl Mech Rev, 47(7):209-226, 1994.

[3] H. Ouyang, W. Nack, Y. Yuan, and F. Cocheteux. Numerical analysis of automotive disc brake squeal: a review. International Journal of Vehicle Noise and Vibration, 1(3):207-231, 2005.

[4] N. Hoffmann, M. Fischer, R. Allgaier, and L. Gaul. A minimal model for studying properties of the mode-coupling type instability in friction induced oscillations. Mechanics Research Communications, 29:197-205, 2002.

[5] G. Fritz, J-J. Sinou, J-M. Duffal, and L. Jezequel. Investigation of the relationship between damping and mode-coupling patterns in case of brake squeal. Journal of Sound and Vibration,307:591-609, 2007.

[6] A. Heussaff, L. Dubar, T. Tison, M. Watremez, and R. F. Nunes. A methodology for the modelling of the variability of brake lining surfaces. Wear, 289:145-159, 2012.

[7] E. Sarrouy, O. Dessombz, and J.-J. Sinou. Piecewise polynomial chaos expansion with an application to brake squeal of linear brake system. Journal of Sound and Vibration, 332:577-594, 2013.

[8] S. Oberst and J.C.S. Lai. Statistical analysis of brake squeal noise. Journal of Sound and Vibration, 330(12):2978-2994, 2011.

[9] F. Massi, L. Baillet, O. Giannini, and A. Sestieri. Brake squeal: linear and nonlinear numerical approaches. Mechanical Systems and Signal Processing, 21(6):2374-2393, 2007.

[10] F. Massi, Y. Berthier, and L. Baillet. Contact surface topography and system dynamics of brake squeal. Wear, 265(11-12):1784-1792, 2008.

[11] J.-J. Sinou. Transient non-linear dynamic analysis of automotive disc brake squeal - on the need to consider both stability and non-linear analysis. Mechanics Research Communications, 37(1):96-105, 2010 .

[12] N. Coudeyras, S. Nacivet, and J.-J. Sinou. Periodic and quasi-periodic solutions for multiinstabilities involved in brake squeal. Journal of Sound and Vibration, 328(4):520-540, 2009.

[13] S. Oberst and J.C.S. Lai. Chaos in brake squeal noise. Journal of Sound and Vibration, 330(5):955$975,2011$.

[14] S. Oberst, J. C. S. Lai, and S. Marburg. Guidelines for numerical vibration and acoustic analysis of disc brake squeal using simple models of brake systems. Journal of Sound and Vibration, $332(9): 2284-2299,2013$.

[15] K. Soobbarayen, S. Besset, and J.-J. Sinou. Noise and vibration for a self-excited mechanical system with friction. Applied Acoustics, 74(10):1191-1204, 2013. 
[16] K. Soobbarayen, S. Besset, and J-J. Sinou. A simplified approach for the calculation of acoustic emission in the case of friction-induced noise and vibration. Mechanical Systems and Signal Processing, 50:732-756, 2015

[17] S. Besset and L. Jezequel. Dynamic substructuring based on a double modal analysis. Journal of Vibration and Acoustics, 130(1):011008, 2008.

[18] D. Brizard, S. Besset and L. Jezequel. Determinantal method for locally modified structures. Application to the vibration damping of a space launcher. Computational Mechanics, 50:631-644, 2012.

[19] R. J. Craig and M.C. Bampton,. Coupling of substructures for dynamic analyses. American Institute of Aeronautics and Astronautics Journal, 6-7:1313-1319, 1968.

[20] J.-J. Sinou, N. Coudeyras and S. Nacivet. Study of the nonlinear stationary dynamic of single and multi instabilities for disc brake squeal. International Journal of Vehicle Design, 51(1-2):207-222, 2008.

[21] G. Fritz, J-J. Sinou, J-M. Duffal, and L. Jezequel. Investigation of the relationship between damping and mode-coupling patterns in case of brake squeal. Journal of Sound and Vibration, 307:591-609, 2007.

[22] O.N. Kirillov and A. O. Seyranian. The effect of small internal and external damping on the stability of distributed non-conservative systems. Journal of Applied Mathematics and Mechanics, 69(4):529-552, 2005

[23] N. Hoffmann and L. Gaul. Effects of damping on mode-coupling instability in friction induced oscillations. ZAMM Z. Angew. Math. Mech., 83(8):524-534, 2003.

[24] J-J. Sinou and L. Jezequel The role of damping and definition of the robust damping factor (rdfactor) for a self-exciting mechanism with constant friction. Journal of Vibration and Acoustics, 129(3):29-306, 2007.

[25] G. Friz, J-J. Sinou, J-M. Duffal, and L. Jezequel. Effects of damping on brake squeal coalescence patterns - application on a finite element model. Mechanics Research Communications, 34(2):181190, 2007. 


\section{List of Figures}

295

$1 \quad$ Design of the finite element model (a) simplified brake system [15](b) 220 uniformly spaced contact nodes at the frictional pad/disc interface (in red

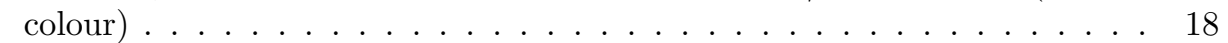

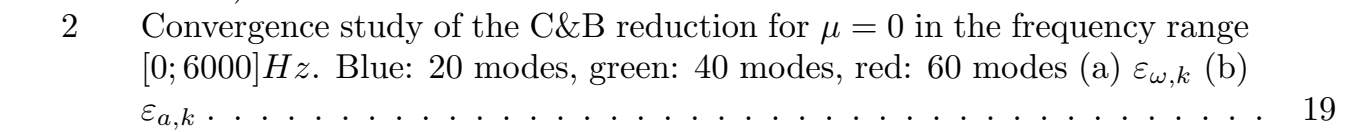

300

3 Convergence study for $\mu=0.5$ (a) $\varepsilon_{\omega, k}^{u}$ (b) $\varepsilon_{a, k}^{u} \cdot-: 1^{\text {st }}$ unstable mode, $\cdot-$ : $2^{\text {nd }}$ unstable mode, - -: $3^{\text {rd }}$ unstable mode. $\ldots \ldots \ldots \ldots \ldots$

4 Convergence study for $\mu=1$ (a) $\varepsilon_{\omega, k}^{u}$ (b) $\varepsilon_{a, k}^{u} \cdot-: 1^{\text {st }}$ unstable mode, $\cdot-$ :

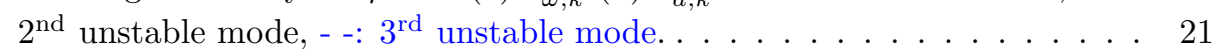

${ }_{305}$

5 Evolution of the real parts for 50,100,150 and 200 generalized degrees of freedom. •: with branch modes, o: without DMS. . . . . . . . . . . 22

convergence for 50,100,150 and 200 generalized degrees of freedom. •:

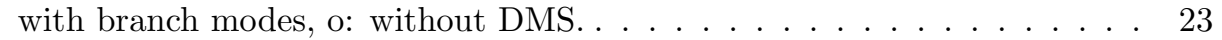

310

7 Evolution of the real and imaginary parts of the second unstable mode convergence for $50,100,150$ and 200 generalized degrees of freedom. •: with branch modes, o: without DMS. . . . . . . . . . . . . 24

8 Evolution of the real and imaginary parts of the third unstable mode convergence for 50, 100, 150 and 200 generalized degrees of freedom. •: with branch modes, o: without DMS. .

315

$9 \quad$ Convergence of the reduced model at $\mu=0.5$.

(a) $\varepsilon_{w k}^{u}($

(b) $\varepsilon_{a, k}^{u}$ unstable mode, $\cdot-: 2^{\text {nd }}$ unstable mode, $--: 3^{\text {rd }}$ unstable mode. $\ldots \ldots .26$

10 Convergence of the reduced model at $\mu=1$. (a) $\varepsilon_{\omega, k}^{u}$ (b) $\varepsilon_{a, k}^{u} \cdot-: 1^{\text {st }}$ unstable mode, $\cdot-: 2^{\text {nd }}$ unstable mode, - -: $3^{\text {rd }}$ unstable mode. $\ldots \ldots .27$

320

11 Computation time: C\&B Vs DMS. Comparison between the two condensation methods increasing the C\&B-order of truncation $[\boldsymbol{\eta}]$ with a fixed number of generalized degree of freedom for the DMS $[\varphi]=100 .\left(\xi_{k}=0.002\right)$.

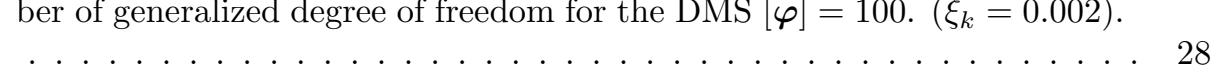




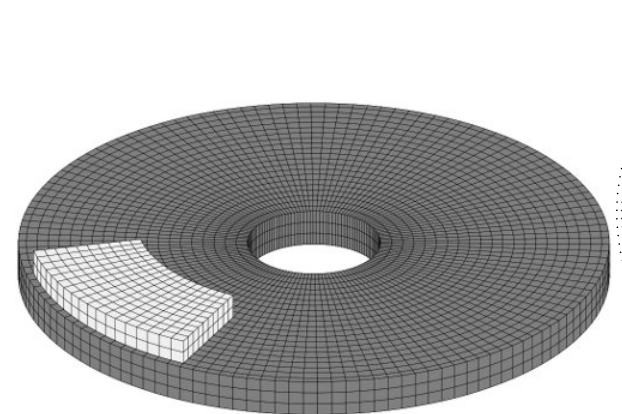

(a)

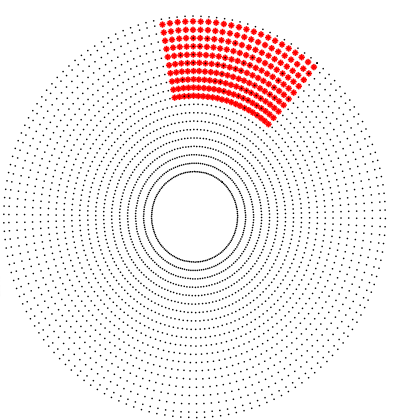

(b)

Figure 1: Design of the finite element model (a) simplified brake system [15](b) 220 uniformly spaced contact nodes at the frictional pad/disc interface (in red colour) 


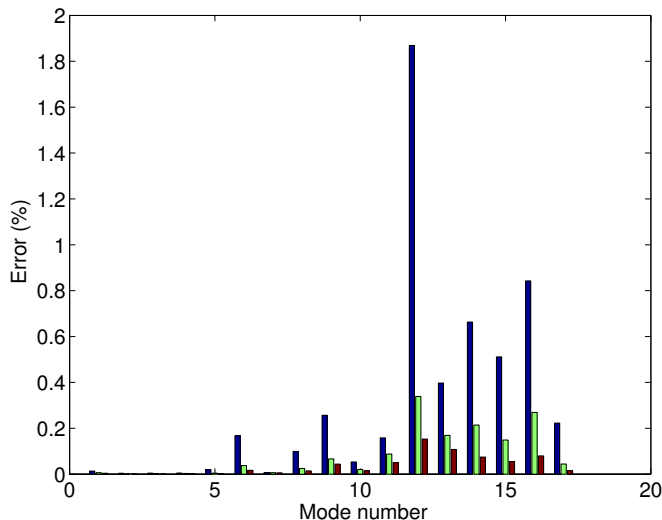

(a)

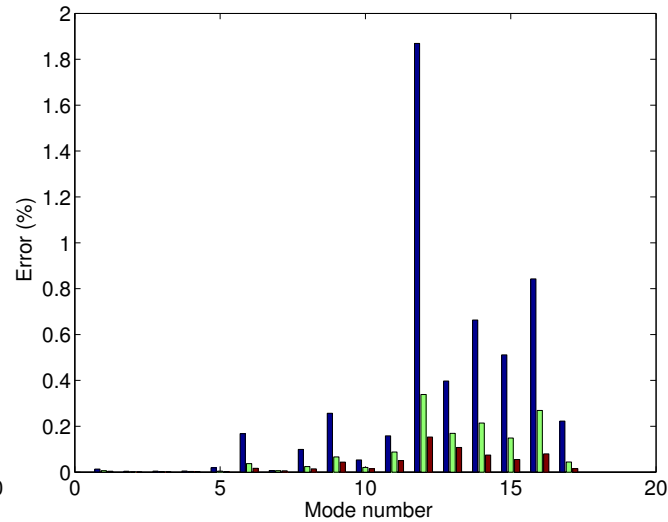

(b)

Figure 2: Convergence study of the $\mathrm{C} \& \mathrm{~B}$ reduction for $\mu=0$ in the frequency range [0;6000]Hz. Blue: 20 modes, green: 40 modes, red: 60 modes (a) $\varepsilon_{\omega, k}$ (b) $\varepsilon_{a, k}$ 


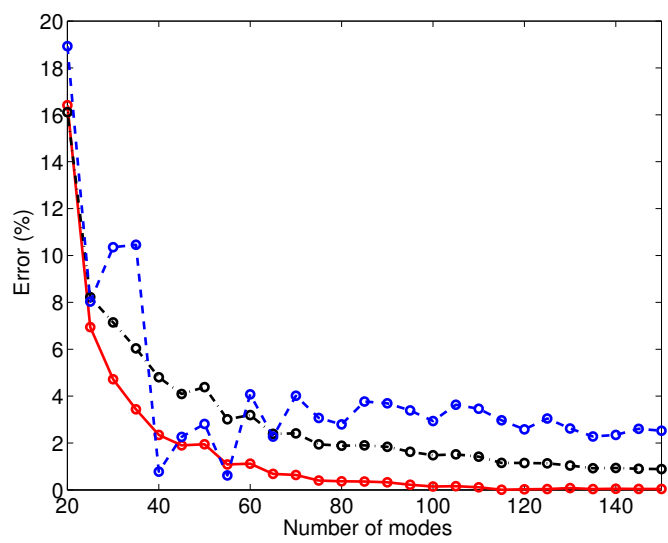

(a)

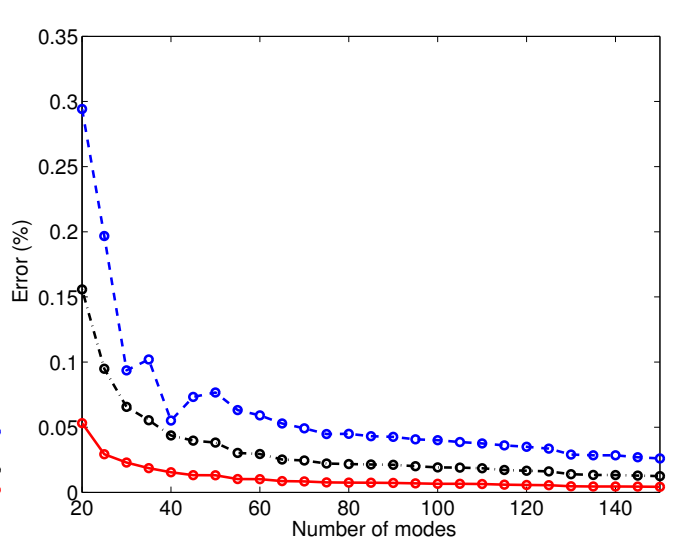

(b)

Figure 3: Convergence study for $\mu=0.5$ (a) $\varepsilon_{\omega, k}^{u}$ (b) $\varepsilon_{a, k}^{u} \cdot-: 1^{\text {st }}$ unstable mode, $\cdot-: 2^{\text {nd }}$ unstable mode, - -: $3^{\text {rd }}$ unstable mode. 


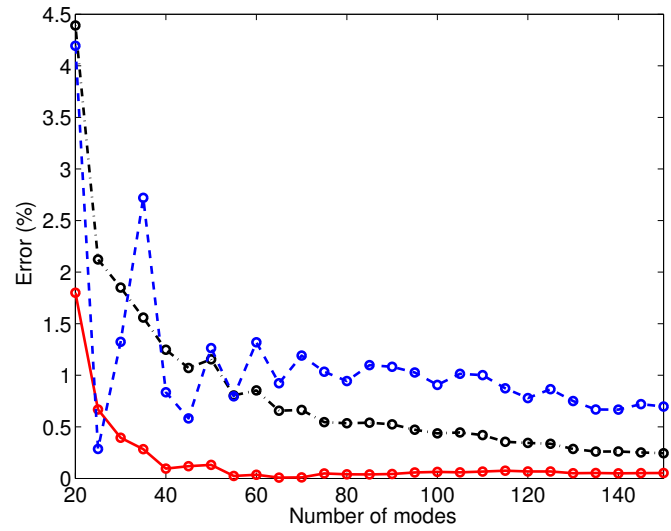

(a)

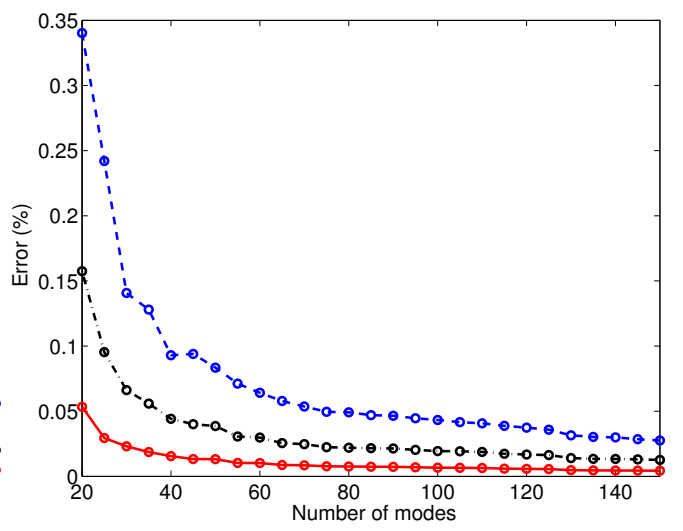

(b)

Figure 4: Convergence study for $\mu=1$ (a) $\varepsilon_{\omega, k}^{u}$ (b) $\varepsilon_{a, k}^{u} \cdot-$ : $1^{\text {st }}$ unstable mode, $\cdot-: 2^{\text {nd }}$ unstable mode, - -: $3^{\text {rd }}$ unstable mode. 

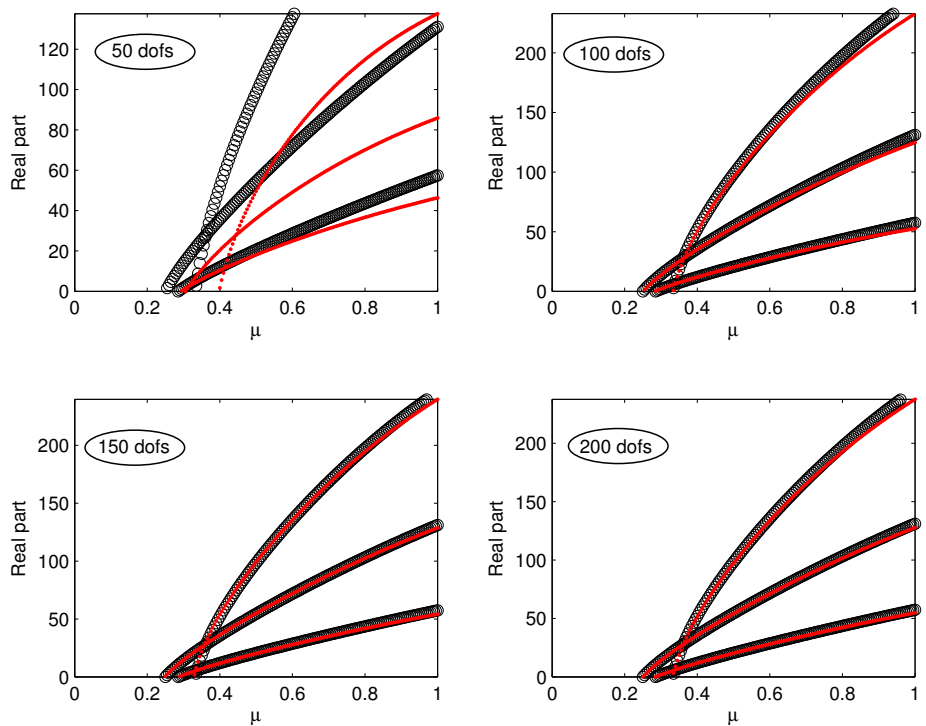

Figure 5: Evolution of the real parts for 50, 100, 150 and 200 generalized degrees of freedom. •: with branch modes, o: without DMS. 

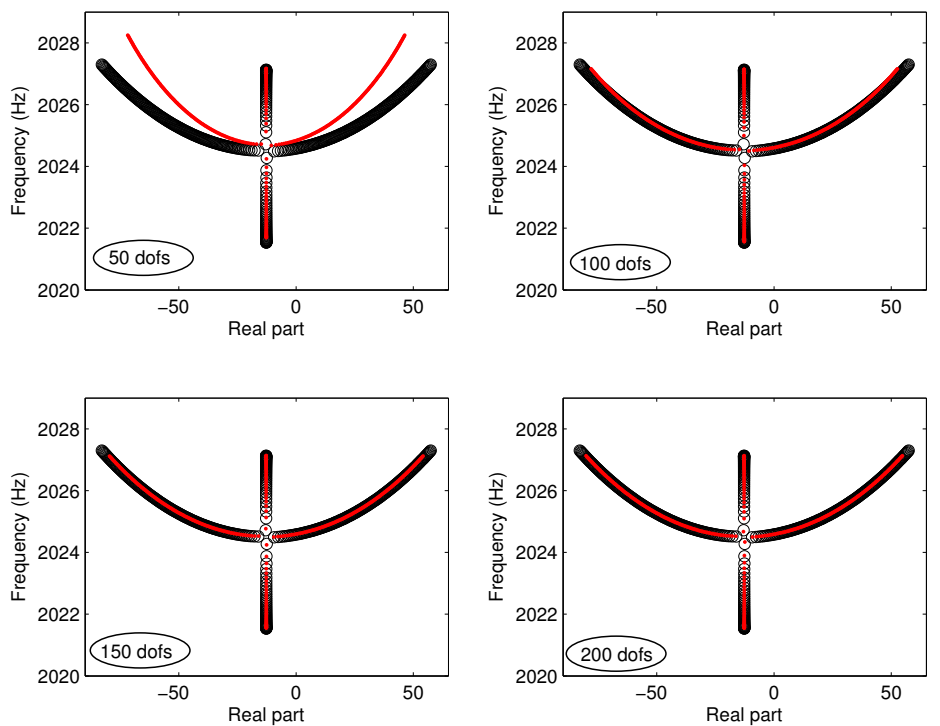

Figure 6: Evolution of the real and imaginary parts of the first unstable mode - convergence for 50,100, 150 and 200 generalized degrees of freedom. •: with branch modes, o: without DMS. 

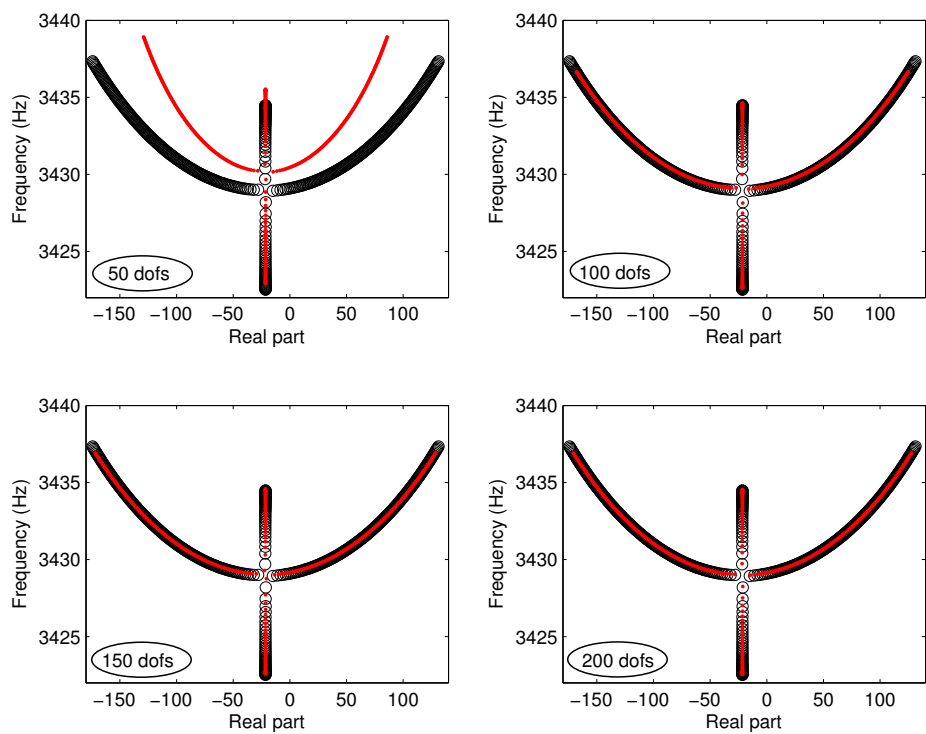

Figure 7: Evolution of the real and imaginary parts of the second unstable mode convergence for 50, 100, 150 and 200 generalized degrees of freedom. •: with branch modes, o: without DMS. 

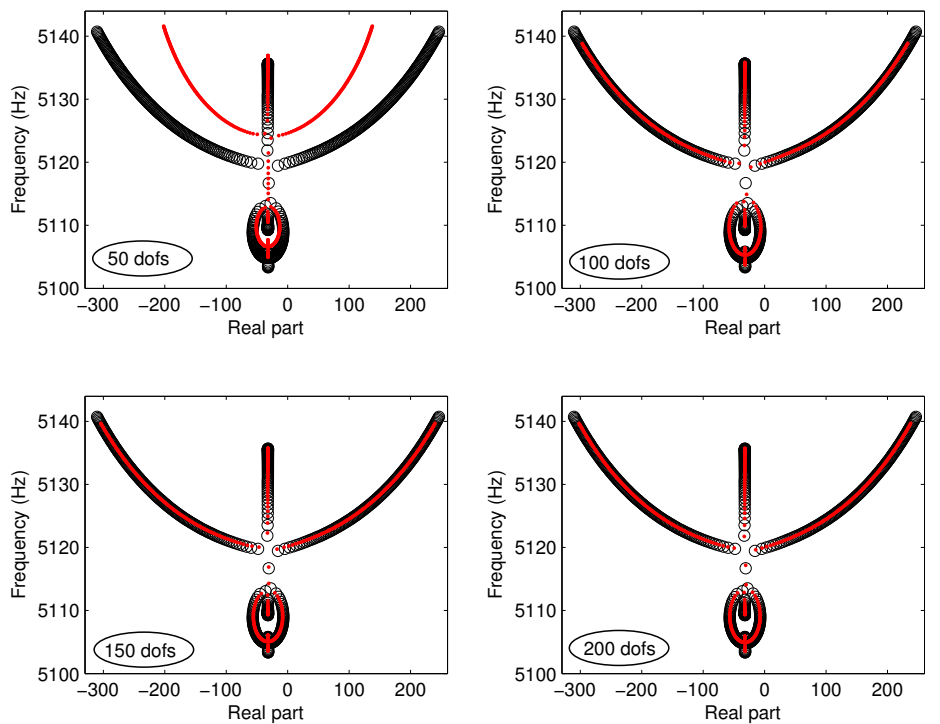

Figure 8: Evolution of the real and imaginary parts of the third unstable mode - convergence for 50, 100, 150 and 200 generalized degrees of freedom. •: with branch modes, o: without DMS. 


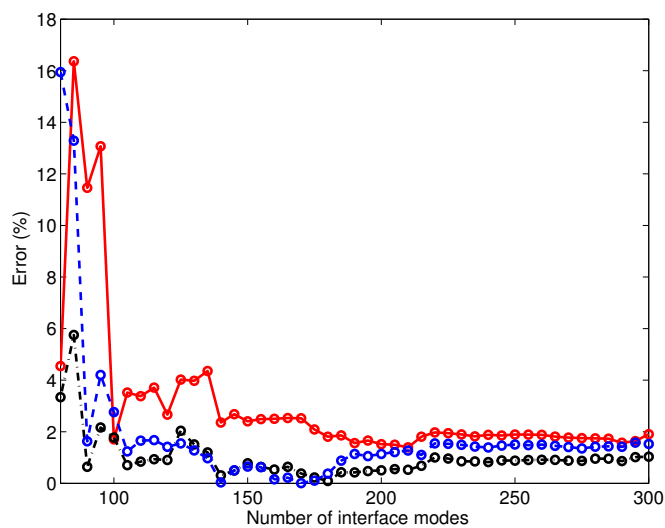

(a)

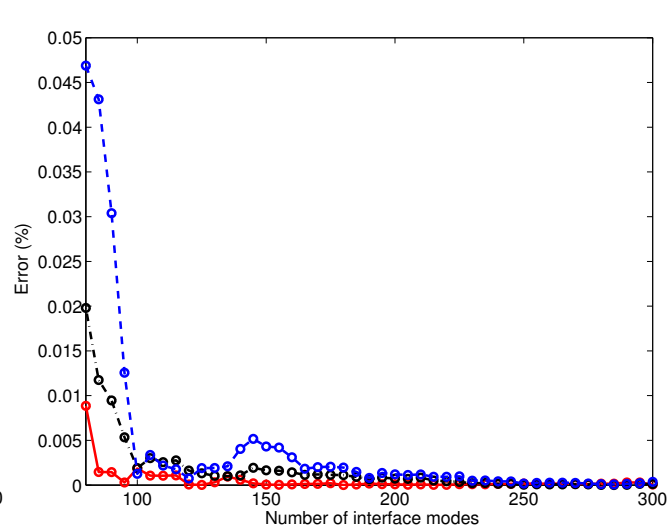

(b)

Figure 9: Convergence of the reduced model at $\mu=0.5$. (a) $\varepsilon_{\omega, k}^{u}$ (b) $\varepsilon_{a, k}^{u}$. -: $1^{\text {st }}$ unstable mode, $\cdot-: 2^{\text {nd }}$ unstable mode, - -: $3^{\text {rd }}$ unstable mode. 


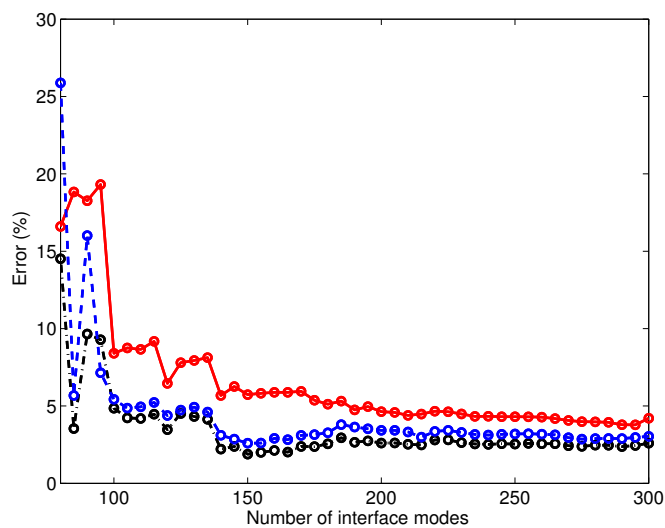

(a)

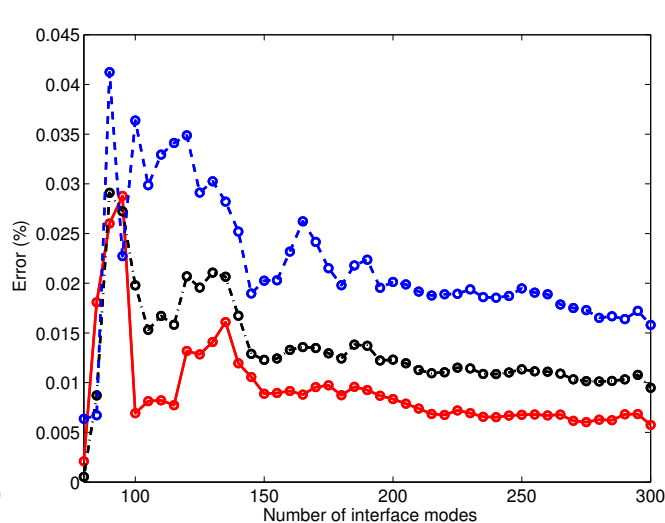

(b)

Figure 10: Convergence of the reduced model at $\mu=1$. (a) $\varepsilon_{\omega, k}^{u}$ (b) $\varepsilon_{a, k}^{u}$. - : $1^{\text {st }}$ unstable mode, $\cdot-: 2^{\text {nd }}$ unstable mode, - -: $3^{\text {rd }}$ unstable mode. 


\begin{tabular}{c|c|c}
\hline $\boldsymbol{\eta}^{P}=\boldsymbol{\eta}^{D}$ & C\&B & DMS $([\boldsymbol{\varphi}]=100)$ \\
\hline 40 & $32.8 \mathrm{~min}$ & $8.2 \mathrm{~s}$ \\
50 & $34.27 \mathrm{~min}$ & $10.3 \mathrm{~s}$ \\
70 & $36.34 \mathrm{~min}$ & $15.7 \mathrm{~s}$ \\
80 & $38.32 \mathrm{~min}$ & $22.7 \mathrm{~s}$ \\
90 & $39.90 \mathrm{~min}$ & $25.8 \mathrm{~s}$ \\
100 & $41.07 \mathrm{~min}$ & $30.99 \mathrm{~s}$ \\
200 & $56.26 \mathrm{~min}$ & $1.8 \mathrm{~min}$ \\
300 & $76.59 \mathrm{~min}$ & $4.6 \mathrm{~min}$ \\
500 & $132.66 \mathrm{~min}$ & $17.17 \mathrm{~min}$ \\
\hline
\end{tabular}

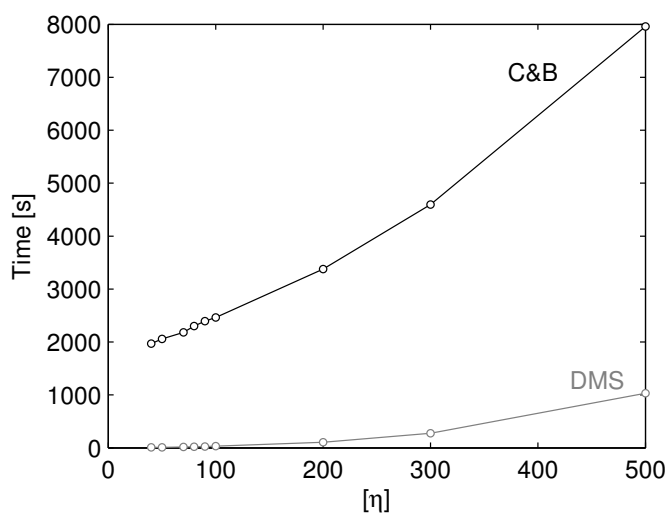

(b)

Figure 11: Computation time: C\&B Vs DMS. Comparison between the two condensation methods increasing the $\mathrm{C} \& \mathrm{~B}$-order of truncation $[\boldsymbol{\eta}]$ with a fixed number of generalized degree of freedom for the DMS $[\boldsymbol{\varphi}]=100 .\left(\xi_{k}=0.002\right)$. 


\section{List of Tables}

$1 \quad$ Finite element details on the simplified brake system . . . . . . . . . . . 30

$325 \quad 2 \quad$ Material and geometric properties of the two substructures . . . . . . . . 31 


\begin{tabular}{l|l|l} 
& Disc & Pad \\
\hline Nodes & 1050000 & 6460 \\
Elements [hexa8] & 114060 & 5860 \\
Dof's & 45000 & 2640 \\
Clamped dof's & 2250 & 440 \\
Matrix size: $[K]=[M]$ & {$[42750 \times 42750]$} & {$[2200 \times 2200]$}
\end{tabular}

Table 1: Finite element details on the simplified brake system 


\begin{tabular}{l|l|l} 
& Disc & Pad \\
\hline Young modulus E [GPa] & 125 & 2 \\
Poisson ratio $\nu[\emptyset]$ & 0.3 & 0.1 \\
Density $\rho\left[\mathrm{kg} / \mathrm{m}^{3}\right]$ & 7200 & 2500 \\
Inner radius $[\mathrm{m}]$ & 0.034 & 0.091 \\
Outer radius $[\mathrm{m}]$ & 0.151 & 0.147 \\
Thickness $[\mathrm{m}]$ & 0.019 & 0.0128
\end{tabular}

Table 2: Material and geometric properties of the two substructures 